\section{Discriminated avoidance and the partial reinforcement effect}

\section{RICHARD D. OLSON*, D. GENE DAVENPORT $\uparrow$, and NEIL C. KAMICHOFF St. Louis University, St. Louis, Mo. 63103}

Groups of 10 female albino rats each received $0 \%, 50 \%$, or $100 \%$ reinforcement followed immediately by the reinterpreted extinction procedure in discriminated avoidance. Performance during acquisition was a direct function of reinforcement percentage, but during extinction performance demonstrated PRE with $100 \%$ Ss significantly below both $50 \%$ and $0 \%$ Ss. Fifty-percent Ss were superior to $0 \%$ Ss during $100 \%$ reinforced reacquisition, but with $0 \%$ Ss better than the Day 1 performance of the $100 \%$ Ss. Frequency distributions of avoidance latency indicated that latency might be further developed as an index of avoidance performance.

Considerable effort has been devoted by learning psychologists to the understanding of the influence of partial reinforcement on both acquisition and extinction of instrumental responding. However, while a number of studies have demonstrated the partial reinforcement effect (PRE) in an instrumental "reward training" situation involving appetitive stimuli such as food (Lewis, 1960), very few have even investigated the PRE in the avoidance paradigm.

Davenport \& Olson (1968) have suggested a modification in the definition of extinction in the discriminated avoidance paradigm, and, more recently, the redefinition has been applied to Sidman avoidance (Davenport, Coger, \& Spector, 1970). The basic argument is that "extinction" involves making a response ineffective in producing the reinforcing consequence that was provided during acquisition. Since the reinforcing consequence crucial for instrumental response acquisition in avoidance conditioning appears to be the termination of the warning signal and/or the omission (or delay) of the shock, it was argued that extinction involves arranging the situation so that the previously effective response no longer results in these consequences. Under the traditional definition, the response is unnecessary because shock is omitted whether or not the response occurs. In the revised definition, the response is ineffective, in that shock is presented whether or not the response is made. The former seems likely to produce a motivational decrease through fear extinction and the latter, instrumental response extinction per se.

The study of partial reinforcement, and in particular the PRE, is influenced by this

*Now at Louisiana State L'niversity at New Orleans, La. 70122.

$\div$ Requests for reprints should be sent to Dr. D. Gene Davenport, Department of Psychology, St. Louis Lniversity. St. Louis, Mo. 63103.

redefinition in two ways. First, partial reinforcement is, contingent upon the occurrence of the instrumental response, the intermittent presentation of reinforcement, and nonreinforcement (extinction) without a cue indicating which condition will be in effect. Thus, the procedure for presenting partial reinforcement is influenced by how extinction is defined. Second, the PRE involves the demonstration that partial reinforcement results in a greater resistance to extinction than that following continuous reinforcement, and the method used to introduce extinction is again crucial for the measure of resistance to extinction.

Davenport, Olson, \& Olson (1970) have reported two investigations of partial reinforcement in the discriminatedavoidance paradigm, using the revised

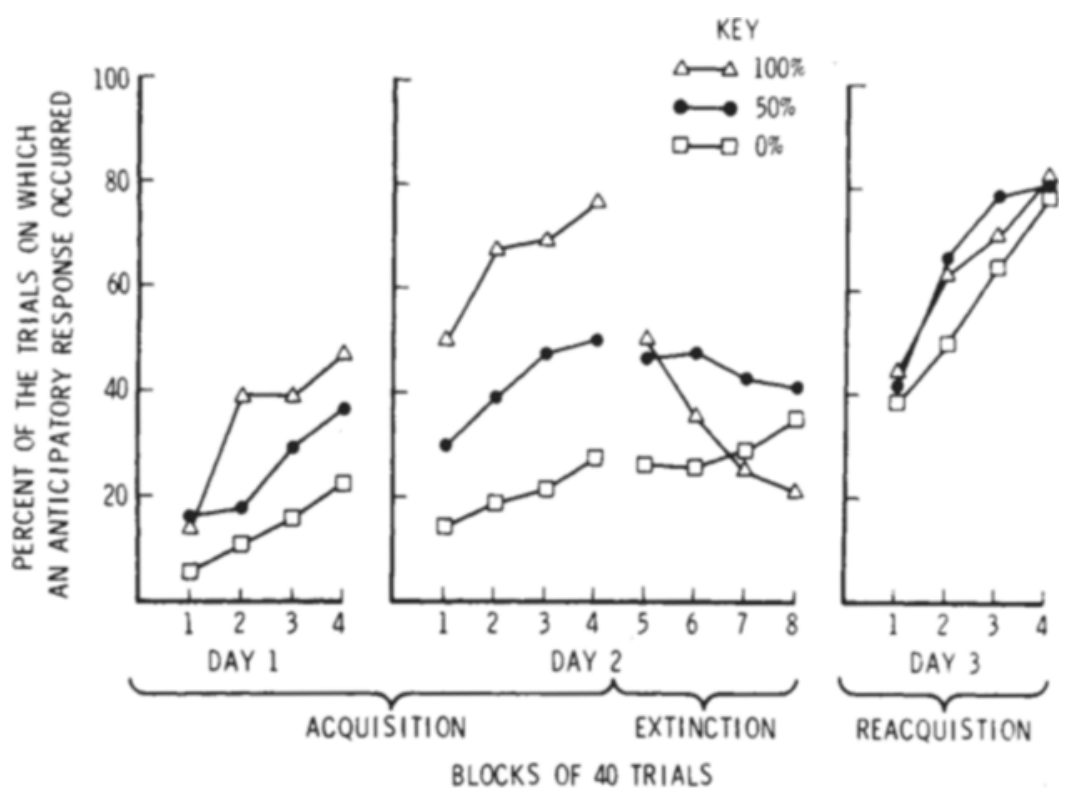

Fig. 1. Mean percent of the trials on which an anticipatory response occurred in blocks of 40 trials for $0 \%, 50 \%$, and $100 \%$ reinforced Ss during acquisition, extinction, and reacquisition $(\mathrm{N}=10)$. reinforcement group.

The purpose of the present experiment was to repeat the crucial aspects of the study by Davenport et al (1970) with a larger sample, naive $S s$, and extinction immediately following acquisition without the 24-h intersession interval. In addition, a reacquisition session was added on the day following the extinction test to determine the effects of 480 trials of escape-only training ( $0 \%$ reinforcement) on the acquisition of avoidance responding.

\section{SUBJECTS}

The Ss were 30 experimentally naive female Sprague-Dawley albino rats, obtained from the colony at the St. Louis University Medical School. All Ss were 92 days old at the start of the experiment and weighed between 170 and $250 \mathrm{~g}$. Ss were maintained with ad lib food and water on a reversed day-night cycle

definition of extinction. They found that acquisition rate and apparently asymptotic performance (at least with 160 trials per day) were a function of the reinforcement percentage. but could find no evidence for the PRE. Results in their second experiment demonstrated a considerably greater "warm-up effect" for the partial reinforcement procedures and suggested that the main experiment (Experiment 1) had used a faulty procedure by beginning extinction on the day following the last acquisition session. Experiment 2 involved immediate extinction but may have suffered from the small sample, extended training, the presence of a prior extinction test, or a need for more training trials per day to overcome "warm-up" in the partial

. 
throughout the study.

\section{APPARATUS}

A standard single-lever Gerbrands operant-conditioning box was used as the testing chamber, with the appropriate programming and recording equipment housed in a separate room. The aversive stimulus was scrambled electric shock from a Lehigh Valley constant-current shocker applied to the grid floor.

\section{PROCEDURE}

Three experimental groups of 10 Ss each were given discriminated avoidance-escape training under $0 \%, 50 \%$, or $100 \%$ reinforcement. For acquisition a leverpress during the 10-sec light buzzer warning signal $\left(\mathrm{S}^{\mathrm{D}}\right)$ terminated the signal and prevented the occurrence of a .4-mA shock on an appropriate percentage of trials; or, if avoidance did not occur, the same response terminated both the $\mathrm{S}^{\mathrm{D}}$ and the shock. During extinction and on nonreinforced trials, the avoidance response was ineffective (i.e. $\mathrm{S}$ could escape but could not avoid). Ss received 2 days of acquisition, were extinguished immediately following the second acquisition session, and then received additional acquisition training on the $100 \%$-reinforcement schedule on the third day. Each session consisted of 160 trials administered with an average intertrial interval of $22.5 \mathrm{sec}$. The study was run in two replications of a five-cycle design, with each cycle consisting of one $S$ per condition in a counterbalanced order.

\section{RESULTS AND DISCUSSION}

The effects of partial reinforcement on both acquisition and extinction are presented in Fig. 1. A 2 (replications) by 3 (reinforcement percentage) by 8 (40 trial blocks) mixed analysis of variance was applied to the percentage of trials on which anticipatory responses occurred during acquisition. The main effect due to reinforcement percentage was significant $(\mathrm{F}=9.11, \quad \mathrm{p}<.01, \mathrm{df}=2 / 24)$. Further comparisons based on Cochran's $t$ (Lindquist, 1953, p. 272) applied to the eighth block of acquisition showed the $100 \%$ group significantly better than the $50 \%$ group $(\mathrm{t}=8.20, \mathrm{p}<.01)$ and that the $50 \%$ was significantly better than the $0 \%$ group $(t=6.62, p<.01)$. In addition, there was a significant effect for blocks $(\mathrm{F}=41.09, \mathrm{p}<.01, \mathrm{df}=7 / 168)$ and for the interaction of Blocks by Reinforcement Percentage $(F=4.88$, $\mathrm{p}<.01$, df $=14 / 168)$. Taken together, this demonstrated that there was an overall increase in percentage of anticipatory responses with practice and that the rate of improvement was a direct function of the percentage of reinforcement. These results were in agreement with those reported by Davenport, Olson. \& Olson (1970).
Two effects of replications were also significant: those involving interactions with blocks $(\mathrm{F}=2.86, \mathrm{p}<.01, \mathrm{df}=7 / 168)$ and those with Blocks by Reinforcement Percentage $\quad(F=1.84, \quad p<.05$, $\mathrm{df}=14 / 168$ ), but not for the main effect of replications. No clear indication is available for the cause of the replication effects, although a check following the completion of the second replication indicated that the shock might have inadvertantly been increased to $.6 \mathrm{~mA}$. The effect on responding was a slower initial rate and a higher overall rate for the $100 \%$ group in the second as compared to the first replication. This appeared to have produced the significant replication jnteractions during acquisition. Replications were not significant in any of the remaining analyses.

To evaluate the reliability of the PRE apparent in Fig. 1, a similar three-way analysis of variance was applied to the last acquisition block and the four extinction blocks of Day 2. There was a significant Blocks effect $\quad(F=14.61, p<.01$ $\mathrm{df}=4 / 96)$, indicating an overall decline in performance due to extinction, and a significant Blocks by Reinforcement Percentage interaction $(F=14.90, p<.01$ $\mathrm{df}=8 / 96)$, suggesting a different rate of decline.

Individual comparisons of the three groups on the last block of extinction showed that the $100 \%$ group was inferior to both the $50 \%$ group $(\mathrm{t}=7.48, \mathrm{p}<.01)$ and the $0 \%$ group $(t=5.14, p<.01)$, with no difference between $50 \%$ and $0 \%$. Thus, dramatic evidence for the PRE is provided, as well as at least weak evidence for a contrast effect (Crespi, 1944), with the $100 \%$ group falling significantly below the $0 \%$ controls when shifted from the "large" to the "small" incentive.

As indicated in the procedure, all animals were provided with $100 \%$ reinforcement on the third day. Two analyses of variance were performed involving these data. First, groups were compared on their performances during this third day. There was evidence of significant effects due to the previous reinforcement percentages ( $F=5.34$, $\mathrm{p}<.05, \mathrm{df}=2 / 24)$, with apparently the $50 \%$ greater than $100 \%$ greater than $0 \%$. However, individual comparisons showed the only significant difference, that of $50 \%$ over $0 \%(\mathrm{t}=2.56, \mathrm{p}<.05)$. The second analysis compared the first day of avoidance acquisition for the $100 \%$ group (which occurred on Day 1) with the first day of avoidance acquisition for the $0 \%$ group (which occurred on Day 3 , having on the two earlier days received 480 escape-only trials). There was a significant superiority of the $0 \%$ over the $100 \%$ group
$(\mathrm{F}=4.93, \quad \mathrm{p}<.05, \quad \mathrm{df}=1 / 16)$ and $\mathrm{a}$ significant blocks effect $(F=14.28$. $\mathrm{p}<.01, \mathrm{df}=3 / 48$ )

These results are rather interesting. First, the superior performance of the $50 \%$ group on Day 3 suggests that not only are the effects of partial reinforcement in slowing extinction demonstrable in terms of responding during extinction, but also may be present in terms of "savings" in the reacquisition of the response. However, in the simple comparison, the superiority of the $50 \%$ over the $100 \%$ was not significant, so it is not possible to make too much of this. Second, the comparisons of the first-day reacquisition for the $100 \%$ and $0 \%$ groups provides no indication of "learned helplessness" (Seligman \& Maier, 1967), although the actual helplessness hypothesis was introduced in the context of inescapable shocks rather than unavoidable shocks. The present evidence suggests that pretsaining with unavoidable but escapable shocks increases the rate of acquisition of the avoidance response if the well-established escape response has become anticipatory. In the present study $0 \%$ Ss were making nearly $40 \%$ of their first responses in the avoidance interval, which dramatically increases the probability of early "reinforcement" by shock omission when avoidance training is begun. There might well be considerable advantage in a procedure which obtained "operant level" baseline performances of all $\mathrm{Ss}$ in an escape-only (0\%) condition for purposes of matching groups on the tendency to make anticipatory responses prior to avoidance training.

Figure 2 provides a summary of the frequency distributions of the latency data in blocks of 40 trials, separately for the three reinforcement groups. The data for the acquisition trials demonstrate very comparable performances for all three groups on the first block of training. Then the $100 \%$ group shows a rapid increase in the number of responses that have latencies at about $2-4 \mathrm{sec}$. This is followed by an increase in anticipatory responding by the $50 \%$ groups, but, in this case, the responses are distributed pretty much throughout the warning interval with no specific peak. A separation of the 50\% group into its two replications proved interesting, with Replication 1 showing a slight peak at the end of the interval (approximately like that of the $0 \% \mathrm{Ss}$ ) and Replication 2 showing a slight peak at the 2- to 4-sec intervals (as shown for the $100 \% \mathrm{Ss}$ ). In neither case was the peak sharp, and the combined data of Fig. 2 does fair justice to both replications. As in previous research from this laboratory, the $0 \%$ group peaked at the end of the warning interval.

As seen in Fig. 2, the effects of 


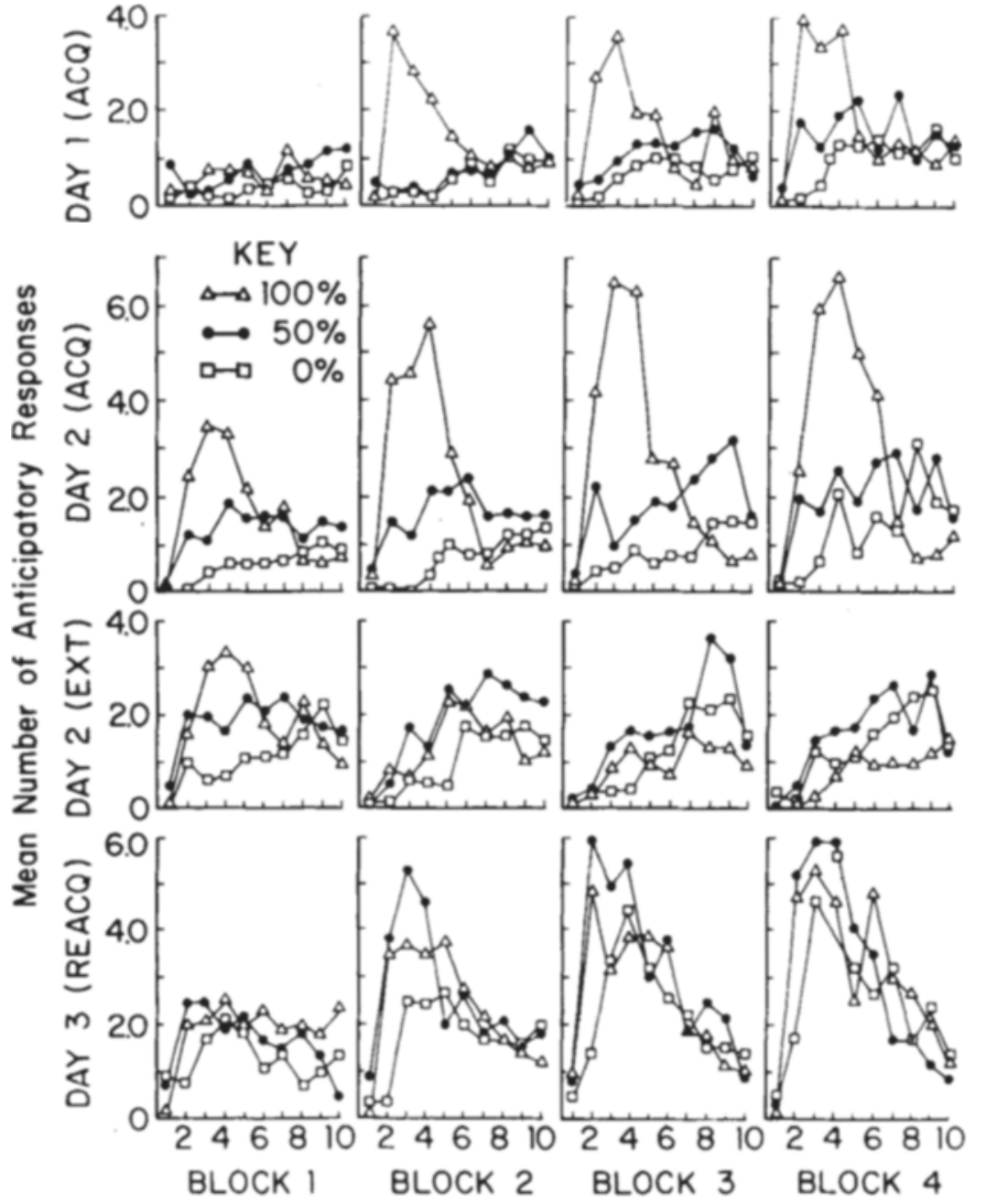

Tenths of Preshock Warning Interval (40 trial blocks)

Fig. 2. Mean latency distributions for the $0 \%, 50 \%$, and $100 \%$ reinforcement Ss in tenths of the warning interval for 40 trial blocks of acquisition, extinction, and reacquisition $(\mathrm{N}=10)$.

extinction on both of the previously reinforced groups was for them to gradually develop a latency distribution which peaked at the end of the warning interval, approximating that of the unreinforced $0 \%$ group. In terms of finer details, the 50\% Ss had a higher overall frequency than did 0\% Ss (especially in the shorter intervals), and the $100 \%$ Ss responded less than the $0 \%$ Ss (especially in the longer latency intervals). In reacquisition, where all of the groups percentage of anticipatory responses. Consistent changes occur for which the average distribution is a rather accurate summary of individual performances. Among these are the shift in peak Iatency shown when extinction is introduced, the variations in the location of the peak under different reinforcement percentages, and the overall shape of the distribution. It may turn out that a good training criterion for avoidance learning will involve matching an individual S's performance in terms of latency distribution with the average distribution from some criterion group. At present the "picture" provided by the frequency distribution seems to us worth a thousand words involving the more restricted traditional statistics, at least until the crucial factors are abstracted out.

The present study provides strong evidence for PRE in the discriminated-avoidance paradigm. The subtle variations produced by manipulations of the reinforcement contingencies in the avoidance situation give us confidence in the essential "operant" nature of the leverpress response in discriminated-avoidance conditioning. In addition, the data seem to provide further indication of the basic similarity between aversive and appetitive training when the appropriate functional correspondences of the crucial variables are identified.

\section{REFERENCES}

CRESPI, L. P. Amount of reinforcement and level of performance. Psychological Review, 1944, 51, 341-357.

DAVENPORT, D. G., COGER, R. W., \& SPECTOR, N. J. The redefinition of extinction applied to Sidman free-operant avoidance conditioning. Psychonomic Science, 1970, 19 , 181-182.

DAVENPORT, D. G., \& OLSON, R. D. A reinterpretation of extinction in discriminated avoidance. Psychonomic Science, 1968, 13, $5-6$.

DAVENPORT, D. G., OLSON, R. D., \& OLSON, G. A. Preliminary analysis of partial reinforcement in discriminated avoidance. Psychonomic Science, 1971, 22, 9-11.

LEWIS, D. J. Partial reinforcement: A selective review of the literature since 1950. Psychological Bulletin, 1960, 57, 1-28.

LINDQUIST, E. F. Design and analysis of experiments in psychology and education. Boston: Houghton-Mifflin, 1953.

SELIGMAN, M. E. P., \& MAIER, S. F. Failure to escape traumatic shock. Journal of Experimental Psychology, 1967, 74, 1-9. 